\title{
Alternative processing during development of a macronuclear chromosome family in Oxytricha fallax
}

\author{
Glenn Herrick, Deborah Hunter, Kevin Williams, and Kenneth Kotter \\ Department of Cellular, Viral and Molecular Biology, University of Utah School of Medicine, Salt Lake City, Utah 84132 USA
}

\begin{abstract}
Macronuclear chromosomes in Oxytricha fallax, a hypotrichous ciliate, are very short. They often belong to small families of cross-hybridizing chromosomes of two or three different sizes. For example, the 81-MAC family consists of three sizes of macronuclear chromosomes $(4.9,2.9$, and $1.6 \mathrm{kbp}$ ) (Cartinhour and Herrick 1984). We show that the family actually consists of two closely related sets of three each and that the two sets are independently created by alternative processing of two separate precursor (micronuclear) versions. Chromosomes of a set share a common 1.6-kbp region, which contains a transcribed gene coding for a 25-kD protein. Different-sized macronuclear chromosomes of a set result from alternative choices of positions for telomere formation. All six members of the family are reproducibly generated in each developing macronucleus, and their copy numbers are stably maintained during vegetative replication of the macronucleus (Herrick et al. 1987). Here we argue for the existence of three distinct copy control elements in the 81-MAC family chromosomes. A model is discussed in which, following polytenization of the micronuclear chromosomes, different chromatids are processed differently, and, subsequently, replication-competent macronuclear chromosome products are amplified under the influence of the vegetative copy control elements.
\end{abstract}

[Key Words: Oxytricha fallax; macronuclear chromosomes; copy control elements]

Received July 9, 1987; revised version accepted September 22, 1987.

Irreversible developmental genome alterations affect essentially every locus in the somatic nucleus of the hypotrichous ciliated protozoa (for review, see Klobutcher and Prescott 1986; Ammermann 1985; Steinbrück 1986). Although each organism is only a single cell, it contains two highly different nuclei with very different roles. The macronucleus directs the vegetative processes of clonal growth, whereas the micronucleus is vegetatively silent and its primary role is to carry the germ line through clonal expansion to the next conjugation. During conjugation, gametic nuclei are exchanged and join to form the zygotic nucleus of the genetically new individual. One mitotic daughter of the zygotic nucleus gives rise to the new macronucleus, and the other becomes the micronucleus. The exconjugant, so equipped, resumes vegetative fission and establishes a clone of cells called a karyonide.

The developing macronucleus undergoes three distinct periods of change. During the first, chromosomes are polytenized. In the second, many DNA sequences are destroyed, including spliced-out internal eliminated sequences (IESs), whereas retained segment ends receive telomeres consisting of blocks of $\mathrm{dC}_{4} \mathrm{~A}_{4}$ repeats. The resulting linear macronuclear chromosomes are extremely numerous $(\sim 20,000$ kinds) and extremely small (heterogeneous, but averaging $\sim 2 \mathrm{kbp}$. Each chromosome analyzed to date bears only a single gene. In the last period of macronuclear development, the chromosomes repli- cate repeatedly, amplifying the contents of the macronucleus to a vegetative level characteristic of a given species and commensurate with the cell's size.

In a survey of randomly chosen Oxytricha fallax macronuclear chromosomes, cloned whole chromosomes (pMAs) were used to probe blots of native macronuclear DNA (Cartinhour and Herrick 1984). We were struck by the high frequency of what were termed "families" of macronuclear chromosomes, the two or three members of which strongly cross-hybridize but differ in size. Subsequently other such families have been discovered, including the $O$. nova $\mathrm{c} 4+\mathrm{c} 5$ chromosome family (Klobutcher et al. 1986), O. fallax histone $\mathrm{H} 3$ and $\mathrm{H} 4$ families (K. Williams and G. Herrick, unpubl.), and an $O$. nova $\mathrm{H} 4$ family (see Discussion). The 81-MAC family, a representative from our initial survey, was chosen for further study (for an example of its blot hybridization pattern, see Fig. 2A). It consists of three different-sized chromosomes, 4.9, 2.9, and $1.6 \mathrm{kbp}$ long. From restriction site mapping (Cartinhour and Herrick 1984) they appear to share a common region of between 1.3 and 1.6 kbp, with the smallest chromosome (MAC III) consisting primarily or entirely of the common region, plus telomeres. The two larger chromosomes, MAC I and II, bear unique 3.3-3.6 and $1.2-1.5 \mathrm{kbp}$ "arms" appended to the right or left, respectively (Fig. 1).

We suggested that the 81-MAC family of chromosomes is generated by alternative DNA processing 
during macronuclear development. However, an unambiguous demonstration of alternative processing was complicated by the existence of more than one sequence version of the family sequences. Cloning of micronuclear and macronuclear DNA's and extensive DNA sequence determinations have led us to conclude that there are three versions of the 81-MAC family sequences in the micronucleus, $\mathrm{vA}, \mathrm{vB}$, and $\mathrm{vC}$ (Herrick et al. 1987). Each version is defined by a set of micronuclear and/or macronuclear DNA clones showing perfect sequence overlaps. The three versions differ from each other primarily by $2.1-4.6 \%$ base substitutions. The genetic relationship between the three versions is unclear; they could be related loci, or, at another extreme, they could be allelic (discussed in Herrick et al. 1987). Whereas all three versions must be represented in the germ line (micronucleus), we have no evidence for vC DNA in the macronucleus. However, as we show here, $\mathrm{vA}$ and $\mathrm{vB}$ chromosomes are present in the macronucleus. Version A chromosomes bear an SphI site in their common regions, whereas $\mathrm{vB}$ chromosomes lack it $\left(S p h I^{+}\right.$vs. SphI ${ }^{-}$, Fig. 1). Maps of cloned vA and vB macronuclear chromosomes (pMAs) relevant to alternative processing are shown in Figure 1 with solid lines; maps of macronuclear chromosomes we demonstrate to exist here are shown with faint lines. Whereas a cloned mi-

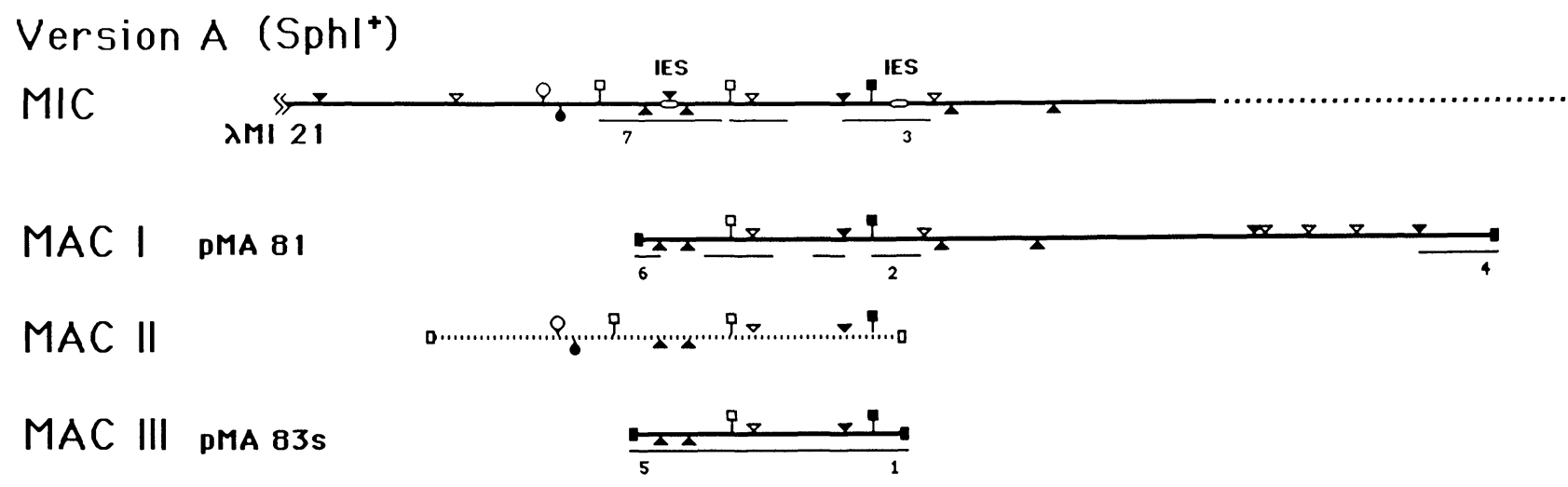

\section{Version $B\left(S p h I^{-}\right)$ \\ MIC}

MAC I

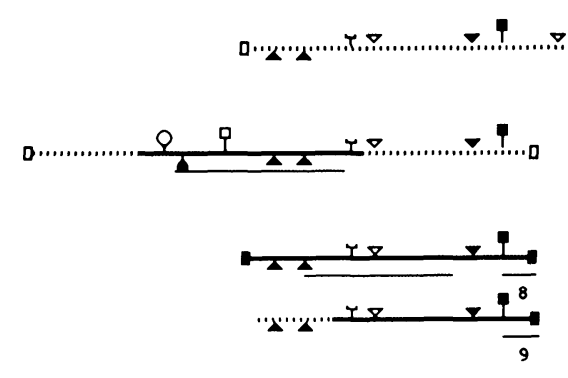

pMA 82d

$Q S+S^{-}$

- $\mathbf{R}$ Y $\mathbf{G}$

○ $\mathbf{O}$ H

- B $1 \mathbf{B}$

$1 \mathrm{kbp}$

Figure 1. Maps of the 81-MAC family chromosomes. Intervals are as deduced by restriction analyses of cloned and uncloned DNAs (Cartinhour and Herrick 1984; Herrick et al. 1985) and adjusted on the basis of sequence information. Maps for DNAs representing versions A (central SphI site present) and B (central SphI site absent) are segregated. For each version the micronuclear map is shown (MIC), followed by the maps of the three macronuclear chromosomes derived from it (MAC I-III). Map regions shown as solid lines have been cloned; the clone names are given at the left. Underlined portions have been sequenced; numbered regions are referred to in the text and in Fig. 3. Macronuclear map regions indicated by broken lines (e.g., version A, MAC II) are deduced from results of blotting experiments (see text). Micronuclear map regions indicated by featureless dotted lines are deduced to exist from the existence of corresponding macronuclear DNAs (see text). Boxes at the ends of macronuclear chromosome maps indicate blocks of telomeric $\mathrm{dC}_{4} \mathrm{~A}_{4}$ repeats (see Fig. 3). Two bubbles in the micronuclear version A map indicate the positions of IESs not present in macronuclear DNA; the leftward portion of this DNA is not shown (full map in Herrick et al. 1985). Restriction site symbols: $(S), S p h I^{\prime}(S)^{-}$, "SphI ${ }^{-"}$ site referred to in the text; $(\mathrm{K}), \mathrm{KpnI}$; (O), XhoI; (B) BamHI; (G), BglII; (H) HindIII; (R) EcoRI. 
cronuclear vA DNA has been obtained ( $\lambda$ MI 21, Fig. 1), no micronuclear vB clones are available, and we only deduce the $\mathrm{vB}$ micronuclear DNA must exist (in some undetermined form) because of the existence of corresponding cloned $\mathrm{vB}$ macronuclear DNAs (discussed in Herrick et al. 1987). It is important to realize that although there are three sequence versions and there are three sizes of macronuclear chromosomes, the two numbers "three" are not related. Specifically, we provide evidence that there are two complete sets of three macronuclear chromosomes each /vA MAC I, MAC II, and MAC III; and vB MAC I, MAC II, and MAC III) and that each set of three chromosomes is generated independently from its corresponding $\mathrm{vA}$ or $\mathrm{vB}$ micronuclear precursor.

Most steps of macronuclear development presumably are invariant. However, some do vary from one developing macronucleus to the next, or even within a given developing macronucleus, as shown in a variety of ciliates both genetically (reviewed by Preer 1969; Bruns 1986) and biochemically (reviewed by Karrer 1986; Austerberry and Yao 1987). For instance, variation in the position of break-telomere addition sites recently has been shown to result in the production of variably sized macronuclear chromosomes (Yao, cited in Yao 1986; Baroin et al. 1987; Forney and Blackburn 1988; Klobutcher et al., in prep.). Such variability in principle can provide somatic flexibility to the species. A special case of variation is that in which the same set of different products of a given germ line sequence is generated reproducibly in each developing macronucleus. Such a situation is analogous to many instances of alternative RNA processing in metazoans wherein the development of the soma leads to a controlled generation of differently processed forms of the same primary transcript in different tissues or times in development (for review, see Breitbart et al. 1987). We adopt the term "alternative processing" to describe the generation of the 81-MAC family of chromosomes because we show that different products of a given micronuclear sequence were generated in a single developing macronucleus, and, furthermore, that the same set of different products is generated each time a new macronuleus develops. To our knowledge this is the first demonstration of alternative processing in any somatic genome alteration system.

\section{Results \\ Different macronuclear chromosomes from the same micronuclear sequence}

In contrast to the numerous differences between versions, scattered every $\sim 20-50 \mathrm{bp}$, the sequenced regions of pMA81 and pMA83s share $704 \mathrm{bp}$ and $1296 \mathrm{bp}$ of perfect homology with $\lambda$ MI 21, respectively, and $866 \mathrm{bp}$ of perfect homology with each other. Thus, we consider these three DNAs to represent a single version (vA). Because pMA81 and pMA83s are independently obtained clones, each of which carries an intact, telomere-to-telomere macronuclear chromosome-a MAC I and MAC III, respectively (Fig. 1) - and both of which are the same version (vA), it is firmly established that vA micronu- clear DNA was processed to give rise to a MAC I chromosome on one hand and a MAC III chromosome on the other. The possibility that any vA MAC II chromosome also was created is left open. Similarly, pMAs $82 \mathrm{~d}$ and 83, which are $\mathrm{SphI}^{-}$(Fig. 1) and differ in sequence considerably from $\mathrm{vA}$ and $\mathrm{vC}$, are perfectly matched in the $221 \mathrm{bp}$ of sequenced overlap. These clones show that the putative $\mathrm{vB}$ micronuclear sequence was processed to generate a vB MAC II and a MAC III chromosome. This one-at-a-time examination of individual, cloned chromosomes is not suited for studying the full composition of the family in total macronuclear DNA, so a different approach was taken.

Version-specific blot hybridization probes were designed and synthesized, based on our knowledge of sequence differences between the versions. Figure 2 compares the results of hybridization of identical, native macronuclear DNA blots with one of two probes, a vBspecific 16-mer (Fig. 2B), and an 18-mer with a sequence shared by all three versions (Fig. 2A). Note first that hybridization and wash conditions were set so that $\mathrm{vA}$ and $\mathrm{vC}$ control DNAs did not hybridize to the $\mathrm{vB}$ probe (lane B2 vs. A2). The vB 81-MAC family has all three sizes of chromosomes, and their relative numbers are in the same proportion as in the family as a whole: MAC III chromosomes predominate, and MAC I and II chromosomes are in roughly equal numbers. With a vA-specific oligomer probe, we have demonstrated a full set of vA MAC I, II, and III chromosomes, present in the same relative proportions (III $>\mathrm{I} \approx \mathrm{II})$, but rarer than the $\mathrm{vB}$ set (not shown).

These blot hybridizations thus confirm what was suggested from the analyses of the cloned DNAs; that is, the 81-MAC family contains two subfamilies, vA $\mathrm{I}+\mathrm{II}+\mathrm{III}$ and $\mathrm{vB} \mathrm{I}+\mathrm{II}+\mathrm{III}$, each produced independently by processing of distinct but highly similar vA and vB precursor sequences.

\section{Processing mechanism}

Figure 3 compares portions of the DNA sequences of the vA MAC I and III chromosomes carried in pMA81 and pMA83s (see maps, Fig. 1). The right subtelomeric sequence of the MAC III chromosome (" 1 " in Fig. 1 ) is colinear with the sequence at the right end of the common region of the MAC I chromosome ("2" in Fig. 1; Fig. 3B) and bears no resemblance to the right MAC I subtelomeric region ("4" in Fig. 1; Fig. 3A). Despite the closely adjoining IES (Fig. 3B), the micronuclear sequence at the latent telomere position (" 3 " in Fig. 1) is colinear with the macronuclear sequences, and these results eliminate direct involvement of the IES in the telomere formation mechanism. This situation is in contrast to a more complex one in Paramecium, where variations in IES processing appear to be involved in variations in the site of telomere formation (Forney and Blackburn 1988). Note also that the left ends of these vA MAC I and III chromosomes are colinear with each other and with the vA micronuclear DNA ("6," "5," and "7" in Fig. 1). The simplest interpretation of these results is that these chromosomes were created by excision of different segments 


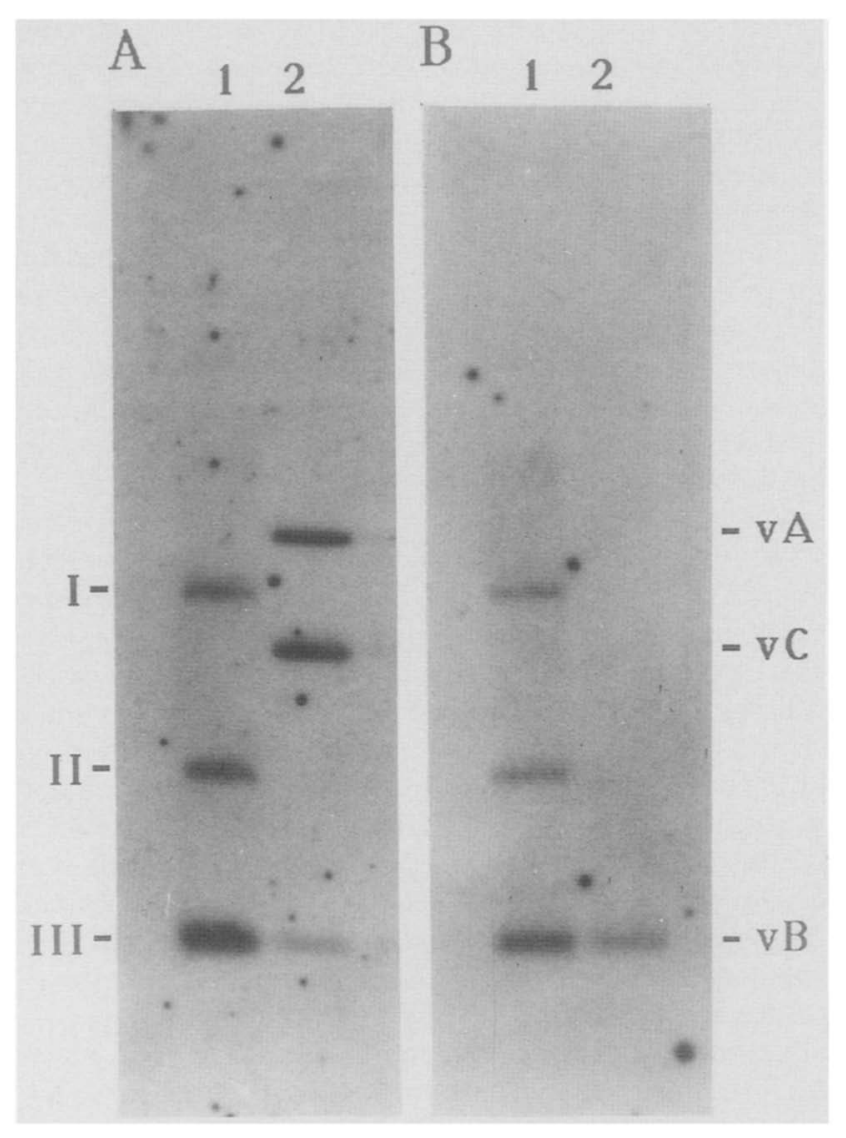

Figure 2. Composition of the 81-MAC family. Native macronuclear DNA and a mixture of control plasmids representing each of the three versions were subjected in duplicate to electrophoresis through a $0.7 \%$ agarose gel and blotted to nitrocellulose. Part $A$ of the blot was hybridized with an oligomer probe with a sequence conserved in all three versions. Part $B$ of the blot was hybridized with an oligomer that perfectly matches vB, but which mismatches with the two other versions. Both oligomers hybridize to areas near the right end of the common region. Lanes $A 1$ and $B 1$ each carry $4 \mu \mathrm{g}$ of macronuclear DNA. Lanes $A 2$ and $B 2$ received equal aliquots of a mix of restriction-cut plasmids bearing each of the three versions of the right end of the common region on different-sized restriction fragments: vA, $0.48 \mathrm{ng}$ of KpnI-digested pMA83s, $5863 \mathrm{bp}$; vC, $0.35 \mathrm{ng}$ of KpnI-digested 22p206 /carries the 0.40kbp KpnI-HindIII fragment of vC micronuclear DNA in pEMBL $8+$ l, $4.40 \mathrm{kbp}$; vB, PstI-digested pMA83, $1.6 \mathrm{kbp}$. The $\mathrm{vA}$ and $\mathrm{vC}$ plasmid amounts were chosen to represent the signal expected in the macronuclear DNA sample if each is present at average copy number (1000/macronucleus); the $\mathrm{vB}$ plasmid DNA was inadvertently underrepresented in the mixture.

from different polytene chromatids and that telomeric repeats were then added. We have not studied the areas involved in the generation of the extreme left and right ends of the family (left MAC II, right MAC I), but we assume they are similar to those at the two ends of the common region. If so, the family would appear to be generated by chromosome breakage variously at four sites, with attendant telomere addition. In contrast to this "excision" model, it is equally possible that the en- tire region is excised by breaks at only two invariant, flanking sites, followed by exonuclease removal of alternative lengths of material before termination with the addition of telomeres. However, for ease of discussion, we will ignore this possibility in favor of simple breakage and telomere addition.

Like Klobutcher et al. (1984) we find no micronuclear $\mathrm{dC}_{4} \mathrm{~A}_{4}$ repeats near the positions of latent telomeres (at "7" and " 3 " in Fig. 1; Fig. 3B, 3D), bolstering the conclusion (Dawson and Herrick 1984; Klobutcher et al. 1984) that all macronuclear telomeres must receive $\mathrm{dC}_{4} \mathrm{~A}_{4}$ de novo, either in concert with or after removal of distal sequences.

The right ends of the common regions of the chromosomes cloned in pMA83 and $\mu 1 \mathrm{Al}$ are not exactly the same but differ by $18 \mathrm{bp}$ in the position of the break-telomere addition events that made them, even though they both are derived from vB sequences (" 8 " vs. "9", Fig. 1; Fig. 3C). [The right end of the vA chromosome of pMA83s is another $5 \mathrm{bp}$ further beyond the $\mu \mathrm{AAl}$ end ("1" vs. "9" Fig. 1; Fig. 3B,C), but this might be attributable to some genetic difference between $\mathrm{vA}$ and $\mathrm{vB}$ ]. Similarly, the two vA left common region ends of pMA83s and 81 chromosomes were created $33 \mathrm{bp}$ apart ("5" vs. "6" Fig. 1; Fig. 3D). These results argue against the simple expectation that single, specific sites at each end of the common region are responsible for the generation of the common region telomeres. Instead they suggest that the target of the process is an area with some diffuse property, as opposed to being a specific short sequence such as recently described in Tetrahymena (Yao et al. 1987). Consistent with this notion, we have examined both the areas surrounding the two common region ends and the latent $\mathrm{c} 2$ chromosome reported for $O$. nova by Klobutcher et al. (1984) and have found no consistent homology with the 15-bp Tetrahymena "chromosome break sequence," nor have we been able to identify any conserved sequence or potential secondary structure shared by the four Oxytricha sites.

\section{Transcription from the 81-MAC family}

A polyadenylated transcript has been detected on RNA blots probed with various portions of the common region (Fig. 4). Its apparent size is $1.33 \times 10^{3}$ nucleotides on both formaldehyde and methylmercury denaturing gels. It is 10-50 times less abundant than the actin transcript we detect with the pOFACT (1.6) probe of Kaine and Spear (1982), and its abundance is not noticeably affected by our usual 2- to 7 -day preharvest $4^{\circ} \mathrm{C}$ incubation of the cells (not shown). Use of complementary singlestranded probes demonstrates that it is transcribed from right to left (not shown). Using a variety of nick-translated subclones as probes (not shown), we have neither detected homology of this transcript with regions outside the common region, nor have we detected any other transcripts from the arms of either MAC I or MAC II, either in total RNA or in polyadenylated RNA preparations. (Since we have no intact MAC II clone, instead we used the $\lambda$ MI $21 B g I I I-E c o$ RI fragment that spans the left end of the common region; see Fig. 1.) 
A. TCTAAGATTGTTAAGATTATGGATAATTCATTTTTGTTTAGAAATATTTTAAATTCAAATTTATAACTACTATAACTACACATGGGGTTTTGGGGTIITGGGGTITTGGGGTITTGGGG TTGATTGCTTATTTTAAAA TAATTTTAATAACTTTATATTAATTATGTTTATTACAAATTATTTCAATTAAGTTCATTCAAAAGGGGTTITGGGGTITIGGGGTITTGGGGTTRTGGGG

B. IES

AATTTTAATAACTTTATATTAATTATGTTTATTACAAATTATTTCAATTAAGTTCATTCAAAAATAAATCTCTGCCACCTTTAAAATCTATATTTAGGTTAACTCAAGTCTTCAAACAA AATTTTAATAACTTTATATTAATTATGTTTATTACAAATTATTTCAATTAAGTTCATTCAAAAATAAATCTCTGCCACCTTTAAAATCTATATTTAGGTTAACTCAAGTCTTCAAACAA AATTTTAATAACTTTATATTAATTATGTTTATTACAAATTATTTCAATTAAGTTCATTCAAAAGGGGTITTGGGGTITTGGGGTITTGGGGTTITGGGG

c. AATTaTAATAACTTTATATTAATTATGTTTATTACAAATTATTTCAATTAAGCTCATTGGGGTITIGGGGTIITGGGGTITIGGGGIITTGGGG AATTaTAATAACTTTATATTAATTATGTTTATTACAAATTGGGGTTTTGGGGTITTGGGGTTTRGGGTTITGGGG

D.

GATTTAGAATGATATATGATATAATGCTTTATAATGTACTTTATCAAAGTAACTTAGGACTGTGATATTAATATCAACTAAGAGTATATATAAATTTAATATTTTAAAATAGGTTMAA CCCCAAAACCCCAAAACCCCAAAACCCCAAAACCCCAAGTAACTTAGGACTGTGATATTAATATCAACTAAGAGTATATATAAATTTAATATTTTAAAATAGGTTTAAA SCCCAAAACCCCAAAACCCCAAAACCCCAAAACCCCAAGAGTATATATAAATTTAATATTTTAAAATAGGTTTAAA

Figure 3. DNA sequences of chromosome ends and common region ends. Sequences are presented $5^{\prime}$ to $3^{\prime}$ as read left to right in the orientation shown in Fig. 1. Telomeric $\mathrm{dC}_{4} \mathrm{~A}_{4}$ and $\mathrm{dG}_{4} \mathrm{~T}_{4}$ repeats are underlined. (A) vA MAC I and III right ends: pMA81 vs. pMA83s; see positions " 4 " and " 1 " of Fig. 1 . (B) vA common region right ends: $\lambda$ MI21, pMA81, pMA83s; see positions " 3 ," " 2, " and " 1 " of Fig. 1. An IES in $\lambda$ MI21 has been removed from display where indicated. $(C)$ vB common region right ends: pMA $\mu 1 A 1$ vs. pMA83; see positions " 9 " and " 8 " of Fig. 1. Nucleotides that differ from vA are lower case. (D) vA common region left ends: $\lambda M I 21$, pMA83s, pMA81; see positions "7", "5," and "6" of Fig. 1.

\section{A protein-coding gene in the common region}

The vA MAC II chromosome of pMA83s was sequenced and was searched for open reading frames, with the assumption that the genetic code used only TGA as "stop," as appears to be the case for ciliates in general (reviewed by Preer 1986). Three long open reading

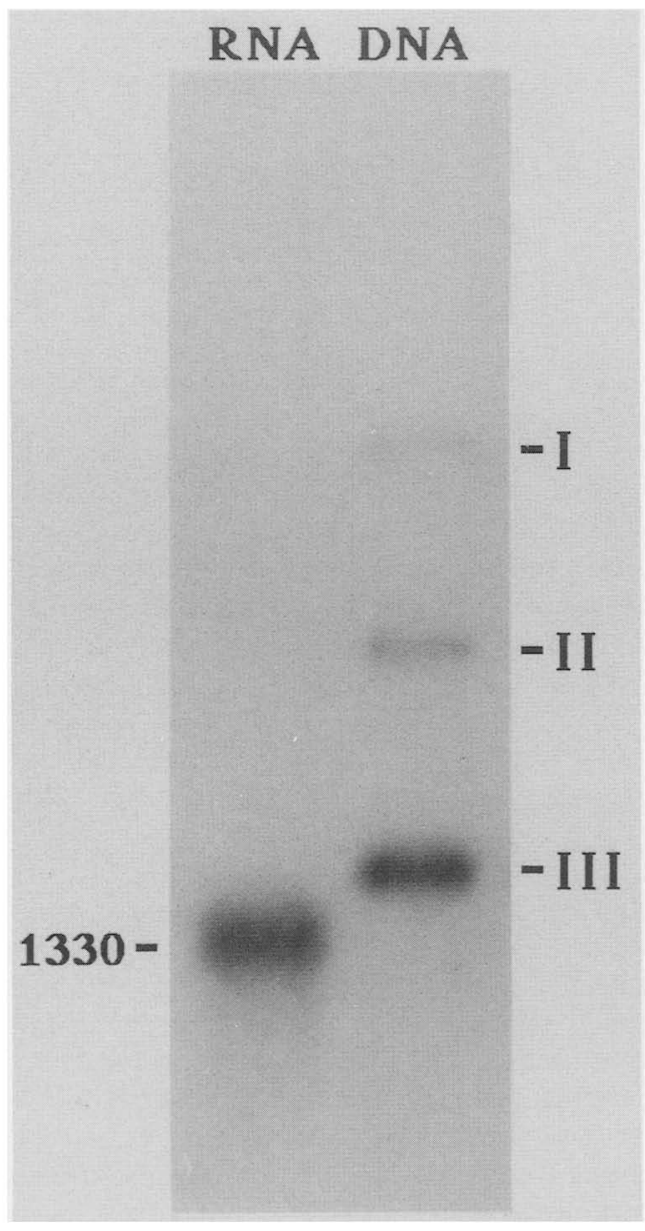

frames were found, all reading from right to left. Their positions are indicated at the bottom of Figure 5. Of these three only the longest appears to be protein coding, as judged by several criteria. First, its codons are minimally diverged from RnY (purine, any base, pyrimidine) over nearly its entire length (Fig. 5, center), a property of genes from a variety of sources (Shepherd 1981), including Paramecium surface antigen genes (Preer 1986). Second, it resides in a relatively GC-rich area (Fig. 5, topl, as is generally true for protein-coding genes in GCpoor genomes (ciliate data reviewed by Karrer 1986). Third, its coding information is almost completely conserved among the three versions, despite numerous nucleotide differences (Fig. 6): that of vB differs at $14 \mathrm{bp}$ from vA but shows no coding differences from $v A_{\text {; }}$ that of $\mathrm{vC}$ differs at $18 \mathrm{bp}$ and shows a single conservative valine-alanine difference. By contrast, the other two vA open reading frames noted are very poorly conserved from version to version (not shown). Fourth, codon usage in the conserved open reading frame is typical of other ciliate genes (see Horowitz et al. 1987). In general, GC-rich codons are avoided: of nine Arg codons, all are AGA and none is AGG or CGN; of 14 Leu codons, none is CTG. In contrast, 11 of $20 \mathrm{Ala}$ codons are GCT, and 10

Figure 4. Northern blot of polyadenylated RNA hybridized with a common region probe. The RNA lane received $0.54 \mu \mathrm{g}$ of polyadenylated RNA isolated from cold-shifted cells. The DNA lane received $25 \mathrm{ng}$ of macronuclear DNA. Electrophoresis was through a $1 \%$ agarose, $2.2 \mathrm{M}$ formaldehyde gel. The probe was prepared from a subcloned HindIII-BgIII fragment from the common region (see Fig. 1); the labeled single strand was not purified for this experiment. For further details, see Experimental procedures. Denatured DNA size standards were run in an adjacent lane, and the apparent size of the RNA was calculated by reference to a linear regression plot of the mobilities of the size standards, the nearest being 1126,1307 , and 1444 nucleotides long. 


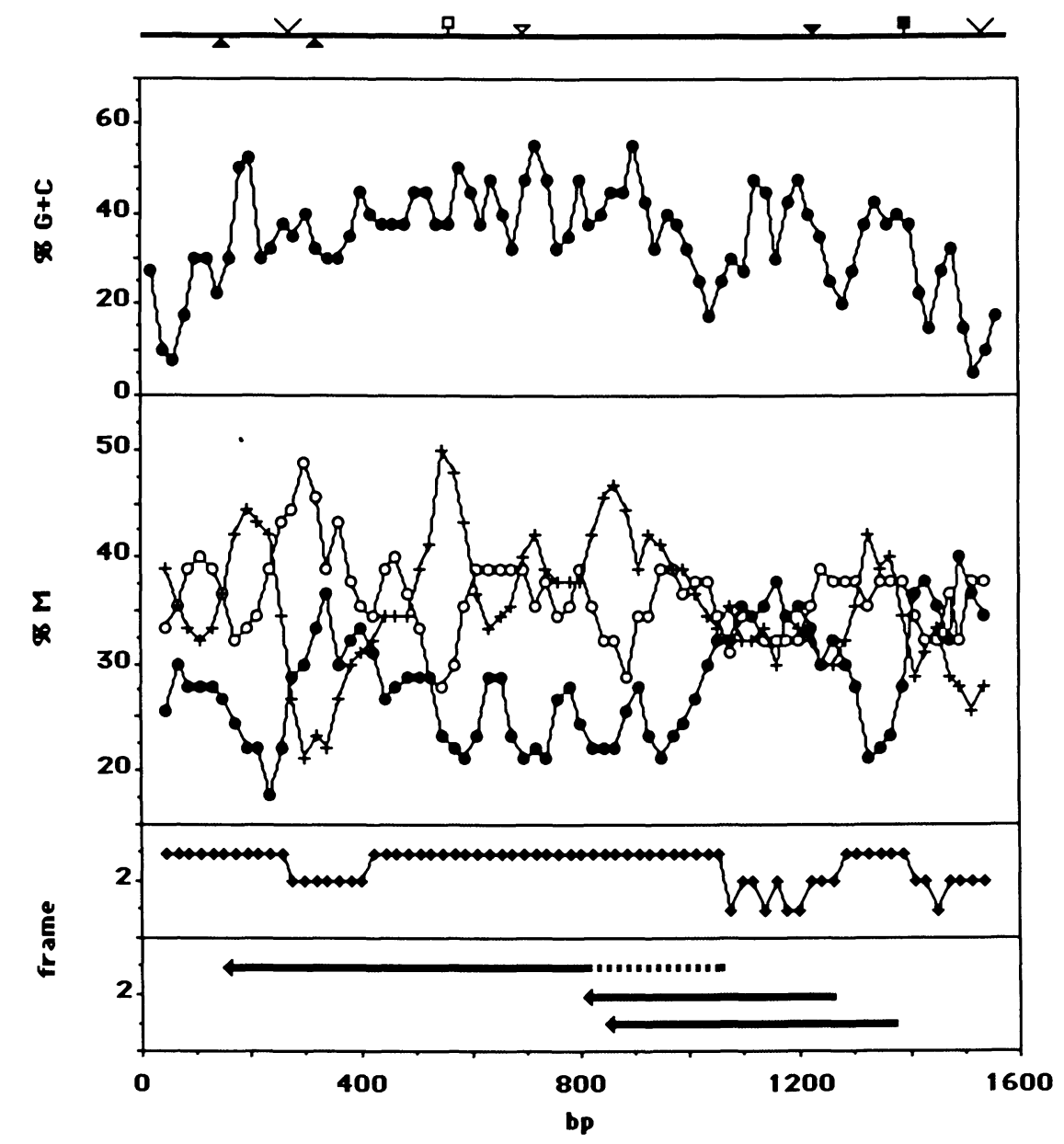

Figure 5. Sequence features of the vA MAC III chromosome from pMA83s. (Top line) Restriction map with carets indicating positions of internal eliminated sequences present only in the micronuclear DNA. Base composition $(\% \mathrm{G}+\mathrm{C})$ : each point represents the average base composition of a window of $40 \mathrm{bp}$, with the window moved $20 \mathrm{bp}$ for each calculation. RnY analysis (Shepherd 1981): percent mutation away from poly $(\mathrm{RnY})$ is plotted for each of the three frames, using a 90-bp window and 21 -bp step. $(0)$ Frame $1 ;(+)$ frame $2 ;(O)$ frame 3 . The top panel labeled "frame" scores the frame with the lowest deviation from poly(RnY) for each interval. The bottom panel shows the positions of the three largest open reading frames; solid arrows indicate ATG-to-TGA intervals, whereas the broken line preceding the ATG in frame 1 is free of TGA stops.

of 18 Gly codons are GGT. Two of nine Gln codons are nonstandard, one TAA and one TAG.

This conserved open reading frame would code for a slightly basic $(B / A=23 / 19)$ 25,407-dalton primary translation product. A search of the National Biomedical Research Foundation (NBRF) protein sequence data base revealed significant homologies with four major, highly conserved regions of metazoan and plant ADP/ATP carrier proteins (Aquila et al. 1982; Arends and Sebald 1984; Baker and Leaver 1985; Adrian et al. 1986). The significance of this finding (not shown) in the context of alternative DNA processing is as yet unclear.

The IES near the left end of the common region $(76 \mathrm{bp}$; Herrick et al. 1987) interrupts the micronuclear counterpart of the gene in such a way that translation would emerge from the IES out of frame and terminate just beyond (Fig. 6). Thus, its precise excision is necessary for production of the functional macronuclear gene, as also is true in a case reported by Klobutcher et al. (1984).

Finally, the 78 codons upstream of the first ATG/Met codon are open. Like the downstream open reading frame, this section is relatively GC-rich and is minimally different from poly(RnY) (Fig. 5). Its 78-residue peptide sequence is completely conserved among the three versions despite several nucleotide differences (Fig. 6). Conceivably, it might be translated in frame with the downstream region if its transcript were spliced to an upstream or a trans exon.

\section{Discussion}

The 81-MAC family chromosomes are generated by alternative processing

The macronuclear DNA analyzed here contains a complete set of three vA and three vB chromosomes. Because $O$. fallax is capable of intraclonal conjugation (although not autogamy) at some stages of clonal life, one might argue that these various $81-\mathrm{MAC}$ chromosomes may not coexist in the same macronucleus. If so, they are not the result of alternative processing within the same macronuclear precursor nucleus, but instead could result from processing variations from one developing macronu- 
cleus to the next (see introduction). However, all macronuclear DNA used in these experiments was obtained from subkaryonide 3.5 , a clone we estabished from a single vegetative cell. Both the parent culture and subkaryonide 3.5 have been senescent and sterile ever since we obtained them. Therefore, we are certain that the various 81-MAC family chromosomes all represent only one developing macronucleus.

Are these various chromosomes generated reproducibly each time a new macronucleus develops? Elsewhere

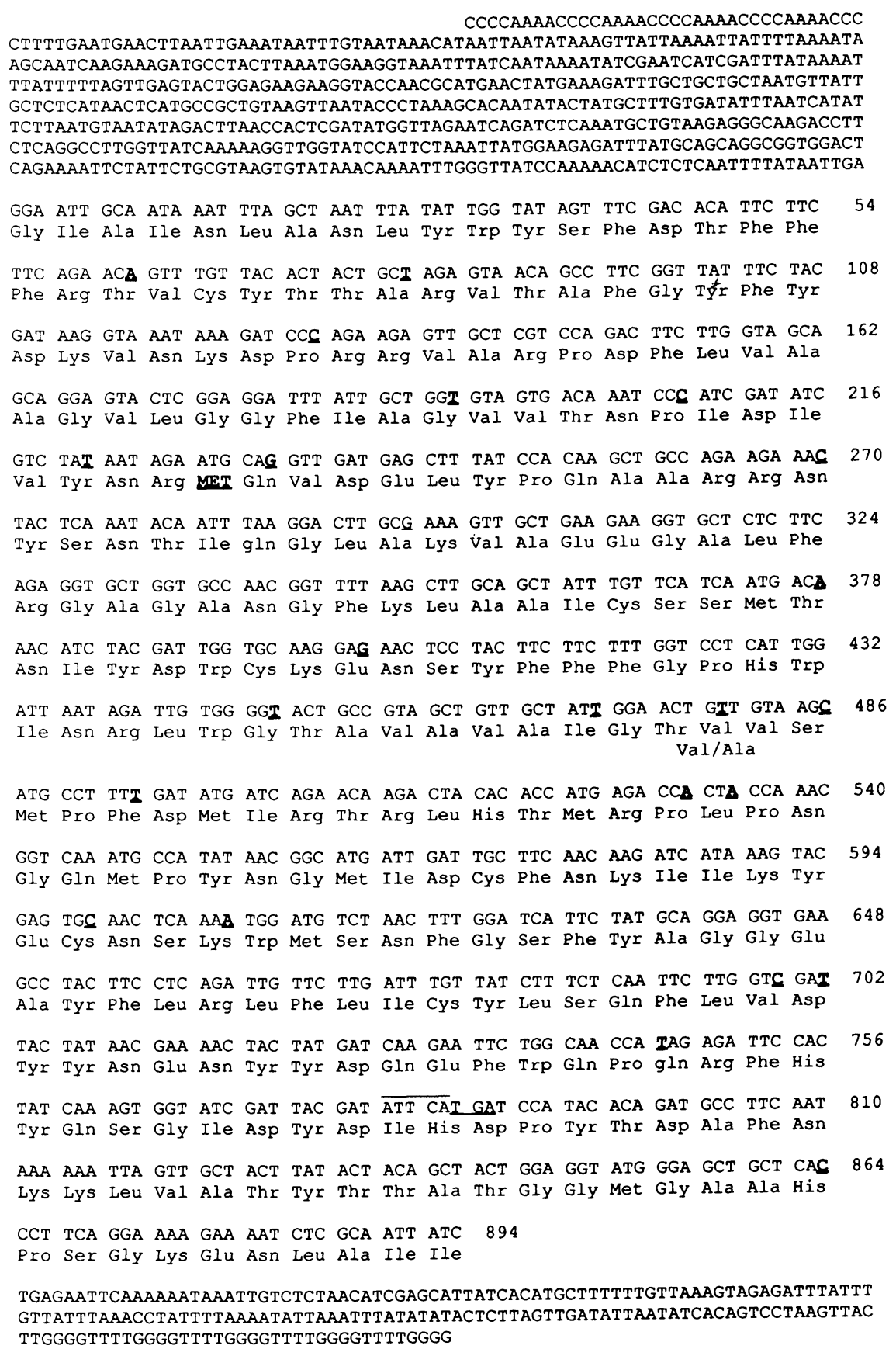

Figure 6. Amino acid conservation of the longest open reading frame of the common region. The entire sequence of the pMA83s insert is shown, with the open reading frame translated (represented in Fig. 5 by the entire arrow in frame 1). The first in-frame ATG is at nucleotide 229, and the first in-frame TGA is at 895 ; corresponding amino acids are shown below each codon; "gln" is assigned to the single TAA and TAG codons at 286 and 745 . The A version is shown. Each underlined, boldface base is different in one or both of the other two versions. In order encountered, the base at the position for each version is listed the vA/vB/vC:A/T/T, T/T/C,C/T/C,T/ $\mathrm{A} / \mathrm{T}, \mathrm{C} / \mathrm{C} / \mathrm{A}, \mathrm{T} / \mathrm{C} / \mathrm{C}, \mathrm{G} / \mathrm{A} / \mathrm{G}, \mathrm{C} / \mathrm{T} / \mathrm{C}, \mathrm{G} / \mathrm{C} / \mathrm{C}, \mathrm{A} / \mathrm{C} / \mathrm{A}, \mathrm{G} / \mathrm{G} / \mathrm{A}, \mathrm{T} / \mathrm{A} / \mathrm{T}, \mathrm{T} / \mathrm{T} / \mathrm{A}, \mathrm{T} / \mathrm{T} / \mathrm{C}, \mathrm{C} / \mathrm{T} / \mathrm{T}, \mathrm{T} / \mathrm{T} / \mathrm{C}, \mathrm{A} / \mathrm{A} / \mathrm{C}, \mathrm{A} / \mathrm{A} / \mathrm{T}, \mathrm{C} / \mathrm{T} / \mathrm{T}, \mathrm{A} / \mathrm{G} / \mathrm{G}, \mathrm{C} / \mathrm{A} / \mathrm{A}, \mathrm{T} /$ $\mathrm{C} / \mathrm{C}, \mathrm{T} / \mathrm{T} / \mathrm{C}, \mathrm{C} / \mathrm{C} / \mathrm{T}$. One amino acid change results, as indicated below the line, in the order vB/vC. The overlined ATTCA (781-785) represents the sequence, present twice in micronuclear DNA, bounding the IES near the left end of the common region (Fig. 1). Note immediately after the ATTCA an out-of-frame TGA stop codon (underlined) at which translation would stop were it to read through the IES (see text). 
we show that the macronuclear DNAs of the young and sexually immature karyonides 9D1 and 9D2 have native and SphI-digested macronuclear DNA blot patterns indistinguishable from each other and from that of subkaryonide 3.5 macronuclear DNA in a side-by-side test (Herrick and Cartinhour 1984; Herrick et al. 1987). Although we have not performed DNA sequencing or version-specific hybridizations with 9D1 and 9D2 DNAs, these results, in conjunction with our thorough analysis of subkaryonide $3.5 \mathrm{DNA}$, very strongly imply that the complete set of three $\mathrm{vA}$ and three $\mathrm{vB}$ chromosomes is reproducibly generated in each developing macronucleus. Finally, the typical pattern of 81-MAC I, II, and III chromosomes has been seen in at least 39 independently isolated, wild karyonides of related Oxytricha species (R.L. Hammersmith, pers. comm.; R.L. Hammersmith and G. Herrick, unpubl.). We thus feel justified to call the 81-MAC family an alternative processing family, because we are confident that the full set of chromosomes is reproducibly generated within each developing macronucleus. Furthermore, each vegetative individual maintains the full set at its disposal (see below). Whether mechanisms have evolved to take advantage of the alternative forms of the 81-MAC family is not known. It is suggestive that even though homology has diverged sufficiently to require lowered stringency of hybridization to detect the family in the wild Oxytricha isolates, nonetheless the alternative processing feature has been fully maintained, since not only are all three chromosome sizes seen, but they are in the same relative stoichiometry we observe in O. fallax. In any case, given the apparent ubiquity of such alternative processing in the formation of the macronucleus, it seems likely that alternative processing does play a role in gene control in at least some alternative processing chromosome families.

Whether the pattern of microheterogeneity of common region ends seen in subkaryonide 3.5 DNA (Fig. 3) is reproduced in each developing macronucleus is unknown; thus, it may not represent alternative processing, but simply high-resolution variability.

\section{Copy control in the vegetative macronucleus}

An important feature of the 81-MAC family is the unequal and strictly maintained copy numbers of its six members during extended karyonidal expansion (Herrick et al. 1987). It is now clear that a specific, active mechanism regulates the number of copies of the Tetrahymena rDNA macronuclear chromosome. One component of the mechanism has been demonstrated genetically; it resides on the chromosome and participates in the partitioning of a fixed, total rDNA chromosome number between competing allelic forms (Larson et al. 1986; Tondravi and Yao 1986). Whether such active mechanisms exist for other macronuclear chromosomes is unknown, and various models of active and passive copy control have been proposed and discussed (see Preer and Preer 1979). However, it seems reasonable provisionally to take the rDNA case as the general para- digm, in the absence of evidence to the contrary. Given this assumption, given our demonstration of strict maintenance of copy number in the 81-MAC family (Herrick et al. 1987), and now given our knowledge of the anatomy of the chromosomes, we can consider the nature and location of distinct sequence elements that presumably participate in the maintenance of the composition of the family.

First, within a version subset MAC IIIs predominate, and the numbers of MAC Is and of IIs are about the same (Fig. 2B shows the vB subset; see also Herrick et al. 1987). We have estimated the relative copy numbers to be $\sim 1: 1: 10$ (MAC I : II : III), although exact values have not been measured. MAC III has only two sequences not found on either MAC I or II. These span the junctions between the subtelomeric regions and the telomeric repeats (Fig. 3B,D); it is possible that these junction sequences are responsible in a positive manner for maintaining the elevated numbers of IIIs, although if so, the picture is complicated by the subtelomeric microheterogeneity. It is simpler to see how the I and II arms might have negative copy control elements. These elements simply might be inert, extra DNA, serving, for instance, to separate the telomere by a critical distance from a second kind of element (replication origin?) in the common region (see below). Alternatively, they might be more conventional, compact units placed at discrete places in each arm.

Second, the vB subset is an estimated 10-fold more numerous than the vA subset (Herrick et al. 1987). Since all members of a version subfamily share only their common regions, the version difference must-within the rDNA paradigm - be caused by some version-specific element within the common region. In summary, in light of the rDNA work, our results suggest the existence of both common-region and arm elements, which would necessarily interact to control chromosome copy number during each vegetative cell cycle.

\section{Generation of the family in the developing macronucleus}

Chromosome breakage and telomere addition take place immediately after chromosome polytenization /see introductory section). We suggest that different chromatids give rise to alternative products produced by different combinations of breaks (Fig. 7). Note that, even with long autoradiography exposures and excellent probes, we have never seen even hints of chromosomes consisting of solely a left or a right arm, or of a common region with both arms (not shown). One explanation could be that chromatid breakage is highly coordinated and only certain combinations of breaks are made.

A simpler model takes into account the idea that control mechanisms exist to adjust the copy numbers of at least the I+II+III subset of the products one expects from a random "partial digest" of chromatids. If we assume that only the common region has an origin of replication, then arm-alone fragments would be lost rapidly by dilution (in contrast to active destruction, which is 


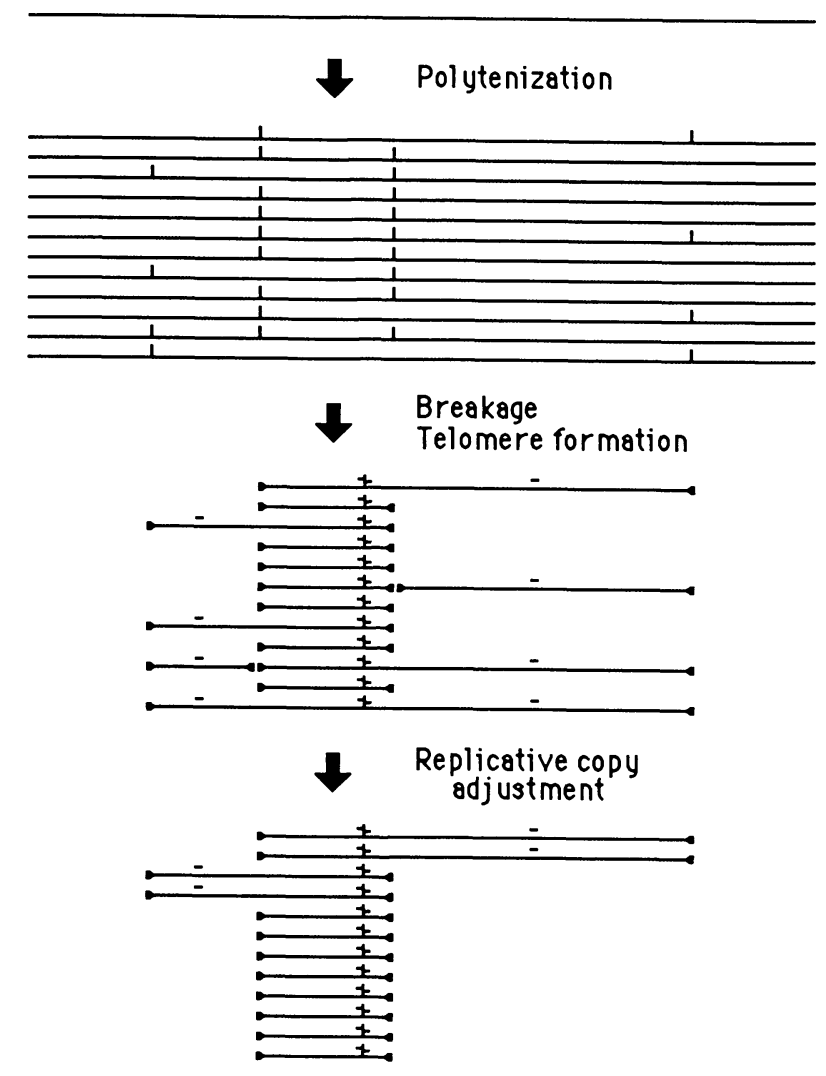

Figure 7. Model for alternative processing of polytene chromatids. See text for explanation. Chromatids are marked where breaks will be made and where telomeres will be added in the second step. $(-)$ The positions of negative copy control elements are represented on the arms. $(+\mid$ An orDNA replication is represented in the common region.

not addressed in this model). Similarly, the absence of chromosomes with a common region and both arms could be a consequence of their bearing a negatively acting copy control element in each arm, leading to a sufficient deficit in replication capacity to cause such chromosomes to be lost by dilution within a few generations, as is the Tetrahymena 11-kbp rDNA chromosome (Pan and Blackburn 1981). Thus, the standard stoichiometry of the three competent chromosomes would become stably established long before a clone has expanded sufficiently to allow biochemical examination of the composition of its family. Presumably, such adjustments would be made already in the several rounds of macronuclear DNA replication that precede emergence of the exconjugant into the vegetative phase (Fig. 7).

An attractive feature of this view is that it provides a possible explanation for the existence of two quite different modes of DNA amplification during macronuclear development. By this view, the polytenization step provides multiple substrates for production of various products, and, if sufficient numbers of chromatids are made, then it becomes statistically unlikely that any of the various products will be omitted from the emerging macronucleus. Having evolved to this state /alternative vs. variable processing), the system is free to evolve mechanisms of differential expression from the alternative chromosomal forms upon which it can depend. The purpose of the second amplification step would be not only to make more copies of the products of the rearrangement step, but also to provide a means for sorting out alternative products and equipping the exconjugant with a mature, copy-adjusted macronucleus competent to drive vegetative processes.

Gene expression from the 81-MAC family chromosomes

To learn what purpose alternative processing might serve, a search of 81-MAC DNA for transcription units and genes was begun. With the possible exception of the long, conserved pre-ATG portion of the conserved open reading frame, our results are consistent with the notion that there is a single gene, solely within the common region, and that the arms are devoid of genes. Of course, our failure to detect other transcripts besides the polyadenylated $\sim 1300$-nucleotide species merely could reflect insensitivity of our techniques; for example, a small or rarely used alternative exon or a tRNA gene could easily have escaped detection. Furthermore, genes expressed only in special conditions would have been missed. However, it is not necessarily unreasonable to consider the idea that the arms are nongenic. Both we $(\mathrm{K}$. Williams and G. Herrick, unpubl., in O. fallax) and R. Stears and C. Jahn (in O. nova; pers. comm.) have detected histone $\mathrm{H} 4$ genes on three sizes of macronuclear chromosomes, reminiscent of the 81-MAC family. The middlesized 1.7-kbp chromosome of O. nova has been cloned and sequenced. The $0.3-\mathrm{kbp} \mathrm{H} 4$ gene is quite close to one end; the bulk of the chromosome is devoid of long open reading frames and GC-poor. Whether the $\mathrm{H} 4$ chromosome family is produced by alternative processing is not known, but one might expect the 81-MAC arms not to have genes. This, of course, need not mean that the arms have no function.

Two kinds of known subtelomeric genes have unusual control features. The case of "expression-linked copies" of trypanosome surface antigen genes is widely recognized (Boothroyd 1985). The surface antigen genes of Paramecium are environmentally regulated and subtelomeric (Preer 1986). The history of the P. tetraurelia mutant d 16 is intriguing (Epstein and Forney 1984). It was selected twice for inability to express its A surface antigen gene, but then reverted to an apparently full wildtype phenotype. Nonetheless, when its DNA was analyzed, the A gene showed an aberrantly processed subtelomeric region, such that the telomere was misplaced immediately $3^{\prime}$ of the gene. This suggests that telomere placement has some effect on the expression of the neighboring gene. Nearly all genes in the macronucleus of a hypotrich might be considered subtelomeric. It seems possible that there is but one gene in the 81-MAC family, located completely within the common region, and that the two subtelomeric arms are involved in some way with differential control of the copies of that gene on the respective chromosomes. We currently are devising experiments to determine from which chromosome(s) the $\sim 1300$-nucleotide RNA is transcribed. 


\section{Experimental procedures}

DNA and RNA isolations

All materials were derived from Oxytricha fallax subclone 3.5. Macronuclear DNA was isolated as described previously /Catinhour and Herrick 1984). All cloned DNAs were isolated previously (Cartinhour and Herrick 1984; Herrick et al. 1985, 1987); each cloned macronuclear DNA (pMA) discussed was isolated independently and shown subsequently to be distinct from each of the others by restriction mapping and/or sequence determination. RNA was isolated by modifications of the procedure of Klobutcher et al. (1984): lysis was in $2 \%$ SDS, $100 \mathrm{mM}$ Tris- $\mathrm{HCl}(\mathrm{pH} 8.1), 10 \mathrm{~mm}$ EDTA, $50 \mu \mathrm{g} / \mathrm{ml}$ potassium polyvinylsulfate, and bentonite was omitted; DNase I (Cooper, DPFF grade) was pretreated with iodoacetate (Zimmermann and Sandeen 1966). Polyadenylated RNA was isolated by chromatography on oligo(dT)-cellulose (Collaborative Research); following denaturation in $15 \mathrm{~mm}$ methylmercury hydroxide, total RNA was diluted 50 -fold into $0.5 \mathrm{M} \mathrm{NaCl}$ and applied to the column at room temperature (Richards and Ehrenfeld 1980). Normally cells were prepared for harvest by a final feeding of autoclaved bacteria, followed by a period of $2-7$ days at $4^{\circ} \mathrm{C}$ to prevent encystment while allowing previous food vacuoles derived from Chlamydomonas to be chased out (Dawson and Herrick 1982). In some cases, RNA was prepared from room temperature-grown cells immediately after they had cleared the final feeding of Chlamydomonas; no significant differences in total or polyadenylated RNA yields were observed: per $10^{6}$ cells, $0.64 \mathrm{mg}$ total RNA, and $5 \mu \mathrm{g}$ polyadenylated RNA $(0.8 \%$ of totall.

\section{DNA sequencing}

Regions to be sequenced were subcloned into one of the pEMBL plasmid vectors, single-strand templates were prepared, and sequences were determined by the dideoxy chain-termination method (Dente et al. 1983; Herrick et al. 1985). The macronuclear chromosome of pMA83s was subcloned in alternative orientations into pEMBL plasmids, and complementary deletion sets were prepared and sequenced by the method of Henikoff (1984). This provided a common region fully sequenced on both strands. Other regions were sometimes sequenced on only one strand when they could be compared to corresponding regions that had been sequenced on both strands. The DNA sequences generated can be obtained from the authors or from GenBank. The DNA sequences corresponding to the marked regions of the clones shown in Figure 1 have the following names and GenBank accession number: SRRHGKH-a, \#13033; mic-KH-a, \#13042; Gp-a, \#13034; pSRRHG-b, \#13031; Kp-b, \#13032. The name indicates the single-letter codes for the landmark restriction sites (see key to Fig. 1) encountered from left to right in the sequence of a version, with the version indicated by the suffix. The sequences are exclusively macronuclear, except for mic-KH-a, which is the vA micronuclear KpnI-HindIII interval carrying the rightward IES. See accession numbers 1302913042 for the full set of sequences relating to the 81-MAC family and Herrick et al. $(1985,1987)$ for descriptions of sequences not described here.

DNA sequences were assembled and manipulated by a variety of computer programs, including RnY analysis (Shepherd 1981) and homology search programs provided by J. Shepherd and run for us by G. Lark, NucFreak (written by E. Kofoid), PC/ Gene, and GEL and SEQ at Intelligenetics (NIH 1 U41:RR01685-05). The Intelligenetics IFIND program was used to search the NBRF protein sequence data base (Release 10).

\section{Gel electrophoresis, blotting, and hybridization}

Southern blotting from agarose gels was as described previously (Cartinhour and Herrick 1984). Northern blots were made from formaldehyde (Goldberg 1980) or methylmercury hydroxide gels (Bailey and Davidson 1976) by capillary transfer onto GeneScreen nylon (DuPont), followed by UV cross-linking (Church and Gilbert 1984).

Southern blot probes were DNA oligomers synthesized as described previously (Herrick et al. 1985). The vB-specific 16-mer 5'-GCAATTTTATAAATCG corresponds to nucleotides 26-41 to the right of the common region $K p n I$ site (see Fig. 1). It mismatches with the corresponding regions of vA ITAAATTTTATAAATCG) and vC (GAAATTTTAGAAATCA). The control 18-mer 5'-GGCATCTTTCTTGATT $\bar{G} C$ exactly matches nucleotides 85-102 to the right of the common region KpnI site in all three versions. Oligomers were $5^{\prime}{ }^{32} \mathrm{P}$-labeled by kination (Zoller and Smith 1984) and used directly as probes. With the 16-mer, prehybridization $(2 \mathrm{hr})$ and hybridization $(20 \mathrm{hr})$ were performed at $37^{\circ} \mathrm{C}$, or $3^{\circ} \mathrm{C}$ below the calculated $T_{d}$ (Suggs et al. 1981), by the protocol of Wood et al. (1985), with 8.4 pmoles of probe. The blot was washed in $6 \times \mathrm{SSC}$ at $4^{\circ} \mathrm{C}$ prior to stringent washes of $1 \mathrm{~min}$ in $6 \times \mathrm{SSC}$ at $41^{\circ} \mathrm{C}$ and $47^{\circ} \mathrm{C}\left(T_{\mathrm{d}}+4^{\circ} \mathrm{C}\right.$ and $T_{\mathrm{d}}+$ $12^{\circ} \mathrm{C}$, respectively). With the 18 -mer, prehybridization $(2 \mathrm{hr})$ and hybridization $(22 \mathrm{hr})$ were performed at $46^{\circ} \mathrm{C}$, by the protocol of Church and Gilbert (1984), with 12.2 pmoles of probe. The blot was washed in $6 \times \mathrm{SSC}$ at $22^{\circ} \mathrm{C}$ prior to a $2.5-\mathrm{min}$ stringent wash in $6 \times \mathrm{SSC}$ at $52^{\circ} \mathrm{C}\left(T_{\mathrm{d}}+6^{\circ} \mathrm{C}\right)$.

Northern blot probes were prepared by a modification of the procedure of Miller (1984) in which $3000 \mathrm{Ci} / \mathrm{mM}^{32} \mathrm{P}$ were incorporated by DNA synthesis across the insert of a single-stranded pEMBL8 subclone, primed by a standard DNA sequencing primer. This product was usually isopropanol-precipitated, denatured, and used directly as a probe. For polarity determinations, the labeled single strand was isolated by restriction cutting at the far side of the insert, electrophoresis in formaldehyde-agrose, and excision of the gel fragment, which was melted and used as a probe. In some cases, nick-translated (Maniatis et al. 1975) plasmids were used as probes. Hybridization was performed at $65^{\circ} \mathrm{C}$ by the procedure of Church and Gilbert (1984), followed by stringent washes in $0.5 \times$ SSC, $0.1 \%$ SDS at $65^{\circ} \mathrm{C}$.

\section{Acknowledgments}

This work was supported by National Institutes of Health grant GM25203. Thanks are due to J. Forney and E. Blackburn, L. Klobutcher, and R. Stears and C. Jahn for communication of unpublished results; to S. Zahler, D. Ammermann, and P. Bruns for useful comments on the manuscript; and to E. Kofoid, J. Shepherd, G. Lark, and B. Condron for generous help with computer analyses.

\section{References}

Adrian, G.S., M.T. McCammon, D.L. Montgomery, and M.G. Douglas. 1986. Sequences required for delivery and localization of the ADP/ATP translocator to the mitochondrial inner membrane. Mol. Cell. Biol. 6: 626-634.

Ammermann, D. 1985. Chromatin diminution and chromosome elimination: Mechanisms and adaptive significance. In The evolution of genome size (ed. T. Cavalier-Smith), pp. 427-442. Wiley, New York.

Aquila, H., D. Misra, M. Eulitz, and M. Klingenberg. 1982. Complete amino acid sequence of the ADP/ATP carrier 
from beef heart mitochondria. Hoppe-Seyler's Z. Physiol. Chem. 363: 345-349.

Arends, H. and W. Sebald. 1984. Nucleotide sequence of the cloned mRNA and gene of the ADP/ATP carrier from Neurospora crassa. EMBO I. 3: 337-382.

Austerberry, C.F. and M.-C. Yao. 1987. Nucleotide sequence structure and consistency of a developmentally regulated DNA deletion in Tetrahymena thermophila. Mol. Cell. Biol. 7: 435-443.

Bailey, J.M. and N. Davidson. 1976. Methylmercury as a reversible denaturing agent for agarose gel electrophoresis. Anal. Biochem. 70: 75-80.

Baker, A. and C.J. Leaver. 1985. Isolation and sequence analysis of a cDNA encoding the ATP/ADP translocator of Zea mays L. Nucleic Acids Res. 16: 5857-5867.

Baroin, A., A. Prat, and F. Caron. 1987. Telomeric site position heterogeneity in macronuclear DNA of Paramecium primaurelia. Nucleic Acids Res. 15: 1717-1728.

Boothroyd, J.C. 1985. Antigenic variation in African trypanosomes. Annu. Rev. Microbiol. 39: 475-502.

Breitbart, R.E., A. Andreadis, and B. Nadal-Ginard. 1987. Alternative splicing: A ubiquitous mechanism for the generation of multiple protein isoforms from single genes. Annu. Rev. Biochem. 56: 467-495.

Bruns, P.J. 1986. Genetic organization of Tetrahymena. In The molecular biology of ciliated protozoa (ed. J.G. Gall), pp. 27-44. Academic, Orlando, Florida.

Cartinhour, S.W. and G.A. Herrick. 1984. Three different macronuclear DNAs in Oxytricha fallax share a common sequence block. Mol. Cell. Biol. 4: 931-938.

Church, G.M. and W. Gilbert. 1984. Genomic sequencing. Proc. Natl. Acad. Sci. 81: 1991-1995.

Dawson, D. and G. Herrick. 1982. Micronuclear DNA sequences of Oxytricha fallax homologous to the macronuclear inverted terminal repeat. Nucleic Acids Res. 10: $2911-2924$.

- 1984. Rare internal $\mathrm{C}_{4} \mathrm{~A}_{4}$ repeats in the micronuclear genome of Oxytricha fallax. Mol. Cell. Biol. 4: 2661-2667.

Dente, L., G. Cesareni, and R. Cortese. 1983. pEMBL: A new family of single-stranded plasmids. Nucleic Acids Res. 11: 1645-1655.

Epstein, L.M. and J.D. Forney. 1984. Mendelian and non-Mendelian mutations affecting surface antigen expression in Paramecium tetraurelia. Mol. Cell. Biol. 4: 1583-1590.

Forney, J.D. and E.H. Blackburn. 1988. Developmentally controlled telomere addition in wild type and mutant paramecia. Mol. Cell. Biol. 8 (in press).

Goldberg, D.A. 1980. Isolation and partial characterization of the Drosophila alcohol dehydrogenase gene. Proc. Natl. Acad. Sci. 77: 5794-5798.

Henikoff, S. 1984. Unidirectional digestion with exonuclease III creates targeted breakpoints for DNA sequencing. Gene 28: $351-359$.

Herrick, G., S. Cartinhour, D. Dawson, D. Ang, R. Sheets, A. Lee, and K. Williams. 1985. Mobile elements bounded by $\mathrm{C}_{4} \mathrm{~A}_{4}$ telomeric repeats in Oxytricha fallax. Cell 43: 759768.

Herrick, G., S.W. Cartinhour, K.R. Williams, and K.P. Kotter. 1987. Multiple sequence versions of the Oxytricha fallax 81-MAC alternate processing family. I. Protozool. 34: 429434.

Horowitz, S., J.K. Bower, G.A. Bannon, and M.A. Gorovsky. 1987. Unusual features of transcribed and translated regions of the histone $\mathrm{H} 4$ gene family of Tetrahymena thermophila. Nucleic Acids Res. 15: 141-160.

Kaine, B.P. and B.B. Spear. 1982. Nucleotide sequence of a ma- cronuclear gene for actin in Oxytricha fallax. Nature 295: 430-432.

Karrer, K.M. 1986. The nuclear DNAs of holotrichous ciliates. In The molecular biology of ciliated protozoa. (ed. J.G. Gall), pp. 85-110. Academic, Orlando, Florida.

Klobutcher, L.A. and D.M. Prescott. 1986. The special case of the hypotrichs. In The molecular biology of ciliated protozoa. (ed. J.G. Gall), pp. 111-154. Academic, Orlando, Florida.

Klobutcher, L.A., C.L. Jahn, and D.M. Prescott. 1984. Internal sequences are eliminated from genes during macronuclear development in the ciliated protozoan Oxytricha nova. Cell 36: 1045-1055.

Klobutcher, L.A., A.M. Vialonis-Walsh, K. Cahill, and R.M. Ribas-Aparicio. 1986. Gene- sized macronuclear DNA molecules are clustered in micronuclear chromosomes of the ciliate Oxytricha nova. Mol. Cell. Biol. 6:3606-3613.

Larson, D.D., E.H. Blackburn, P.C. Yeager, and E. Orias. 1986. Control of rDNA replication in Tetrahymena involves a cisacting upstream repeat of a promoter element. Cell 47: 229-240.

Maniatis, T., A. Jeffrey, and D.G. Kleid. 1975. Nucleotide sequence of the rightward operator of phage lambda. Proc. Natl. Acad. Sci. 72: 1184-1188.

Miller, A.M. 1984. The yeast MATal gene contains two introns. EMBO I. 3: $1061-1065$.

Pan, W.-C. and E.H. Blackburn. 1981. Single extrachromosomal ribosomal RNA gene copies are synthesized during amplification of rDNA in Tetrahymena. Cell 23: 459-466.

Preer, J.R., Jr. 1969. Genetics of the Protozoa. In Research in protozoology (ed. T.-T. Chen) vol. 4, pp. 133-278. Pergamon Press, Oxford.

- 1986. Surface antigens of Paramecium. In The molecular biology of ciliated protozoa. (ed. J.G. Gall), pp. 301-340. Academic, Orlando, Florida.

Preer, J.R., Jr. and L.B. Preer. 1979. The size of macronuclear DNA and its relationship to models for maintaining genic balance. J. Protozool. 26: 14-18.

Richard, O.C. and E. Ehrenfeld. 1980. Heterogeneity of the 3' end of minus-strand RNA in the poliovirus replicative form. I. Virol. 36: 387-394.

Shepherd, J.C.W. 1981. Method to determine the reading frame of a protein from the purine/pyrimidine genome sequence and its possible evolutionary justification. Proc. Natl. Acad. Sci. 78: 1596-1600.

Steinbrück, G. 1986. Molecular reorganization during nuclear differentiation in ciliates. Results Prob. Cell Differ. 13: $105-174$.

Suggs, S.V., T. Hirose, T. Miyake, E.H. Kawashima, M.J. Johnson, K. Itakura, and R.B. Wallace. 1981. Use of synthetic oligodeoxyribonucleotides for the isolation of specific cloned DNA sequences. In Developmental biology using purified genes. (ed. D.B. Brown), pp. 683-693. Academic, New York.

Tondravi, M.M. and M.-C. Yao. 1986. Transformation Tetrahymena thermophila by microinjection of ribosomal RNA genes. Proc. Natl. Acad. Sci. 83: 4369-4373.

Wood, W.I., J. Gitschier, L.A. Lasky, and R.M. Lawn. 1985. Base composition-independent hybridization in tetramethylammonium chloride: A method for oligonucleotide screening of highly complex gene libraries. Proc. Natl. Acad. Sci. 82: 1585-1588.

Yao, M.-C. 1986. Amplification of ribosomal RNA genes. In The molecular biology of ciliated protozoa (ed. J.G. Gall), pp. 179-202. Academic, Orlando, Florida.

Yao, M.-C., K. Zheng, and C.-H. Yao. 1987. A conserved nu- 
Herrick et al.

cleotide sequence at the sites of developmentally regulated chromosomal breakage in Tetrahymena. Cell 48: 779-788.

Zimmermann, S.B. and G. Sandeen. 1966. The ribonuclease activity of crystallized pancreatic deoxyribonuclease. Anal. Biochem. 14: 269-277.

Zoller, M.J. and M. Smith. 1984. Oligonucleotide-directed mutagenesis: A simple methods using two oligonucleotide primers and a single-stranded DNA template. DNA 3: 479488. 


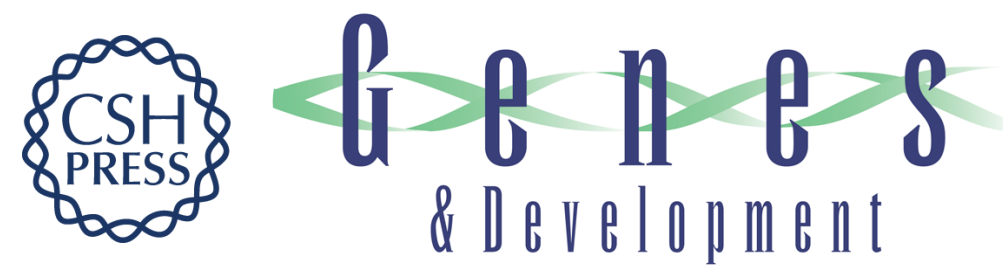

\section{Alternative processing during development of a macronuclear chromosome family in Oxytricha fallax.}

G Herrick, D Hunter, K Williams, et al.

Genes Dev. 1987, 1:

Access the most recent version at doi:10.1101/gad.1.10.1047

References This article cites 36 articles, 13 of which can be accessed free at: http://genesdev.cshlp.org/content/1/10/1047.full.html\#ref-list-1

License

Email Alerting

Receive free email alerts when new articles cite this article - sign up in the box at the top Service right corner of the article or click here.

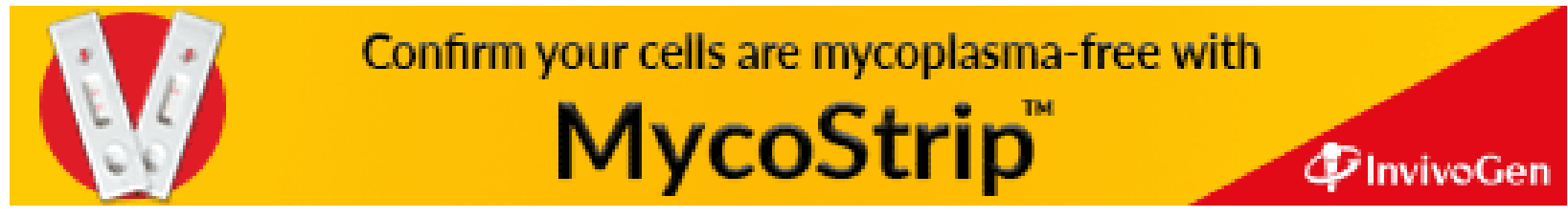

